\title{
A TEST STATISTIC FOR HIGH RESOLUTION POLARIMETRIC SAR DATA CLASSIFICATION
}

\author{
Pierre FORMONT ${ }^{1,2}$, Jean-Philippe OVARLEZ ${ }^{1,2}$, Frédéric PASCAL ${ }^{2}$, Gabriel VASILE $^{3}$ and Laurent FERRO-FAMIL ${ }^{4}$ \\ 1 : French Aerospace Lab \\ ONERA DEMR/TSI - Chemin de la Hunière, 91761 Palaiseau Cedex, FRANCE \\ Tel: +33169936 313 - Fax: +33 169936269 - Email: pierre.formont@ supelec.fr \\ 2 : SONDRA Research Alliance \\ Plateau du Moulon, 3 rue Joliot-Curie, 91192 Gif-sur-Yvette Cedex, FRANCE \\ Tel: +33169851804 - Fax: +33169851809 \\ 3 : Grenoble-Image-sPeech-Signal-Automatics Lab, CNRS \\ GIPSA-lab DIS/SIGMAPHY, Grenoble INP - BP 46, 38402 Saint-Martin-d'Hères, FRANCE \\ Tel: +33476826424 - Fax: +33476574790 \\ 4 : Institut d'Electronique et de Télécommunications de Rennes, CNRS \\ IETR, Campus de Beaulieu, 263 avenue du Général Leclerc, 35042 Rennes Cedex, FRANCE \\ Tel: +33223235224 - Fax: +33223236969
}

\begin{abstract}
Modern SAR systems have high resolution which leads the backscattering clutter to be non-Gaussian. In order to properly classify images from these systems, a non-Gaussian noise model is considered: the SIRV model. A statistical test of equality of covariance matrices is used to classify pixels, taking into account the critical region of the test which rejects the likeliness of a covariance matrix to any of the class centers. This test is applied on experimental data obtained with the ONERA RAMSES system in X-band. The results show a good separation between natural and man-made areas of the image.
\end{abstract}

Index Terms - Image classification, polarimetry, synthetic aperture radar, statistics

\section{INTRODUCTION}

The polarimetric clutter obtained with recent SAR systems tends to have non-Gaussian characteristics. One of the most general and elegant non-Gaussian noise model is provided by the so-called Spherically Invariant Random Vectors (SIRV). This paper proposes a method for classification of high resolution polarimetric SAR data, based on a statistical test of equality of covariance matrices. It is organized as follows : Section 2 presents the statistical framework. Section 3 describes the proposed method. Then, results on experimental data are presented in Sect. 4.

\section{STATISTICAL FRAMEWORK}

A SIRV [1] is a compound Gaussian process defined as the product of a multivariate circular Gaussian process and a scalar random variable:

$$
\mathbf{k}=\sqrt{\tau} \mathbf{x}
$$

where $\tau$, a positive random variable, called texture, whose Probability Density Function (PDF) is unknown and $\mathbf{x}$ is a complex circular zero-mean Gaussian $m$-vector with covariance matrix $\mathbf{T}=E\left[\mathbf{x x}^{H}\right]$, called speckle where $E[$.$] denotes$ the statistical expectation. For POLSAR data, the polarimetric diversity is modeled by the speckle $\mathrm{x}$ containing the 3 polarization channels $H H, H V$ and $V V$, i.e. $m=3$ and the random variation of the power from cell to cell corresponds to the texture $\tau$.

To ideally estimate the covariance matrix of a pixel $(i, j)$, represented by its target vector $\mathbf{k}$, one needs several realizations of the pixel at different times. As it is impossible for 
SAR images, a spatial neighborhood of the pixel $\left(\mathbf{k}_{\mathbf{1}}, \ldots, \mathbf{k}_{\mathbf{N}}\right)$ is required for the estimation process. To test the equality of a population covariance matrix, $\mathbf{T}$ and a known matrix, $\mathbf{T}_{\omega}$, the classical hypothesis test is defined as:

$$
\left\{\begin{array}{l}
H_{0}: \mathbf{T}=\mathbf{T}_{\omega} \\
H_{1}: \mathbf{T} \neq \mathbf{T}_{\omega}
\end{array}\right.
$$

In the classical Gaussian model, the Maximum Likelihood Estimator (MLE) of the covariance matrix is called the Sample Covariance Matrix (SCM):

$$
\widehat{\mathbf{T}}_{S C M}=\frac{1}{N} \sum_{i=1}^{N} \mathbf{k}_{i} \mathbf{k}_{i}^{H}
$$

Under SIRV assumption, the covariance matrix can be estimated thanks to ML theory. Considering a deterministic texture, Gini et al. derived in [2] the exact ML estimate, solution of the implicit equation:

$$
\widehat{\mathbf{T}}_{F P}=\frac{m}{N} \sum_{i=1}^{N} \frac{\mathbf{k}_{i} \mathbf{k}_{i}^{H}}{\mathbf{k}_{i}^{H} \widehat{\mathbf{T}}_{F P}^{-1} \mathbf{k}_{i}}
$$

Existence and uniqueness of the above equation solution $\widehat{\mathbf{T}}_{F P}$, the Fixed Point (FP) estimate, have been investigated in [3], and its statistical properties (consistency, unbiasedness and asymptotic Gaussianity) have been studied in [4]. In practice, it is obtained by the associated recursive algorithm which converges whatever the initialization (see for details [3]).

\section{M-DISTANCE}

In [5], Lee et al. proposed an algorithm to classify pixels in a polarimetric SAR image, based on the statistical distribution of the covariance matrix. This algorithm used a distance measure, called the Wishart Distance, derived from the Wishart distribution of the covariance matrices. We propose to use a novel distance measure derived from a statistical test of equality of covariance matrices, the Box's M-test[6]. As discussed in [7], the test, originally developed for the real case, can be extended to the complex case. The test statistic is given by :

$$
t=\frac{\left|\widehat{\mathbf{T}}_{1}\right|^{\frac{\nu_{1}}{2}}\left|\widehat{\mathbf{T}}_{2}\right|^{\frac{\nu_{2}}{2}}}{\left|\widehat{\mathbf{T}}_{t}\right|^{\nu_{t}}}
$$

where $\nu_{i}$ is the degree of freedom of $\widehat{\mathbf{T}}_{i}, \nu_{t}=\nu_{1}+\nu_{2}$ and $\widehat{\mathbf{T}}_{t}$ is the pooled sample covariance matrix defined by:

$$
\widehat{\mathbf{T}}_{t}=\frac{\nu_{1} \widehat{\mathbf{T}}_{1}+\nu_{2} \widehat{\mathbf{T}}_{2}}{\nu_{1}+\nu_{2}}
$$

As the exact distribution of $t$ is rather cumbersome, Box proposes the following approximation for the distribution of $u$

$$
u=-2\left(1-c_{1}\right) \ln (t) \sim \chi^{2}\left(\frac{1}{2} m(m+1)\right)
$$

where $c_{1}=N\left(\frac{2 m^{2}+3 m-1}{12(m+1)}\right)$ and $\chi^{2}(a)$ denotes the $\chi^{2}$ distribution with $a$ degrees of freedom. This approximation holds for Wishart-distributed matrices. Pascal et al. proved that the FP estimate is asymptotically Wishart-distributed with $\nu=(m / m+1) N$ degrees of freedom in [4]. The $\chi^{2}$ approximation can therefore be used for the FP estimate.

We use the test statistic $u$ from Eq. (3) as a distance measure in the following algorithm:

1. Initially classify the image into 8 areas using the $H / \alpha$ decomposition.

2. Select the first class of the $H / \alpha$ decomposition (the class situated in the top-right corner of the $H / \alpha$ plane) as the first class of the classifier. We have found that chosing a different class for the initialization had little to no influence on the end result.

3. For each pixel, compute the M-distance between its covariance matrix and each class center using Eq. (3) (in the first iteration, there is only one class center).

4. If the minimum distance is lower than the threshold given by $\chi^{2}\left(0.999, \frac{1}{2} m(m+1)\right)$, classify the pixel in the corresponding class. Else, put the pixel in the rejection class.

5. Once all pixels are classified, define the rejection class as a new class and compute its class center.

6. Repeat until there are 8 classes and a rejection class.

\section{APPLICATION ON EXPERIMENTAL DATA}

Experimental data were acquired in X-band by the ONERA RAMSES system in the area of Brétigny, France, with a spatial resolution of approximately 1.5 meter in range and azimuth, and a mean incidence angle of $30^{\circ}$. Fig. 1 is a color representation of the Brétigny area in the Pauli basis : $\mathrm{H} \mathrm{H-}$ $V V$ in red, $H V$ in green and $H H+V V$ in blue. One can clearly distinguish two large buildings on the left side of the image, a parking lot on the right side, two small buildings in the middle, an urban area on the top-left corner and 4 bright spots on the lower-right corner, corresponding to trihedral corners. Fields and forested areas constitute most of the remaining part of the area. The algorithm from Sect. 3 has been applied on these data. The results can be seen below, on Fig. 2.

Fig. 2(a) show the results of the classical Wishart Classifier on our data, using the SCM. While some areas are well separated from the rest (namely, the fields in yellow, green and blue), the buildings areas contain many classes. Fig. 2(b) shows the results of the classifier after the first iteration. There are only 2 classes : the first class of the classifier in blue and 


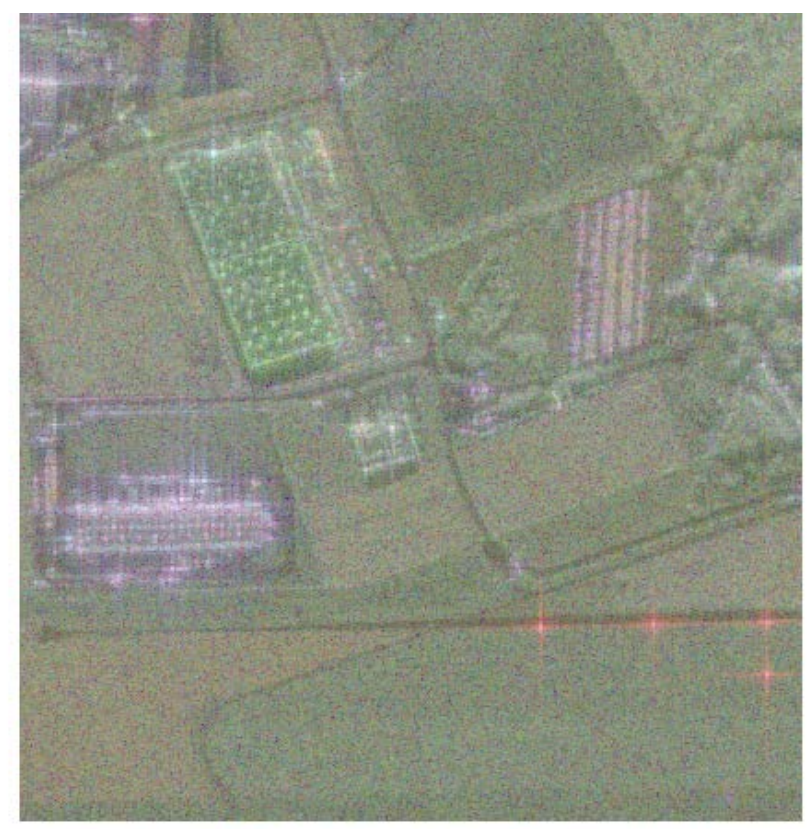

Fig. 1. Color representation of the Brétigny area, France in the Pauli basis : $H H-V V$ in red, $H V$ in green and $H H+$ $V V$ in blue.

the rejection class in brown. The features mentioned above are, for the most part, clearly identifiable on this image and they all belong to the rejection class. Fig. 2(c) shows the results of the classifier after 8 iterations. There are 9 classes, including the rejection class. The number of pixels contained in the rejected class has been reduced from $46 \%$ to $23 \%$. When comparing Fig. 2(b) and Fig. 2(c), the rejected class in brown, although reduced, is still a big part of the image. Regarding the other classes in Fig. 2(c), it is rather difficult to provide a direct subjective interpretation.

\section{CONCLUSION}

In this paper, authors proposed a novel distance measure to classify polarimetric covariance matrices taking into account the critical region inherent in the definition of a statistical test. Results of the classical Wishart Classifier have been compared to our algorithm. The results show a good separation between natural and man-made parts of the scene but further classification is not sufficient and should be improved, e.g. with a pre-segmentation of the image.

\section{REFERENCES}

[1] Kung Yao, "A representation theorem and its applications to spherically-invariant random processes," IEEE Transactions on Information Theory, vol. 19, no. 5, pp. 600-608, 1973.

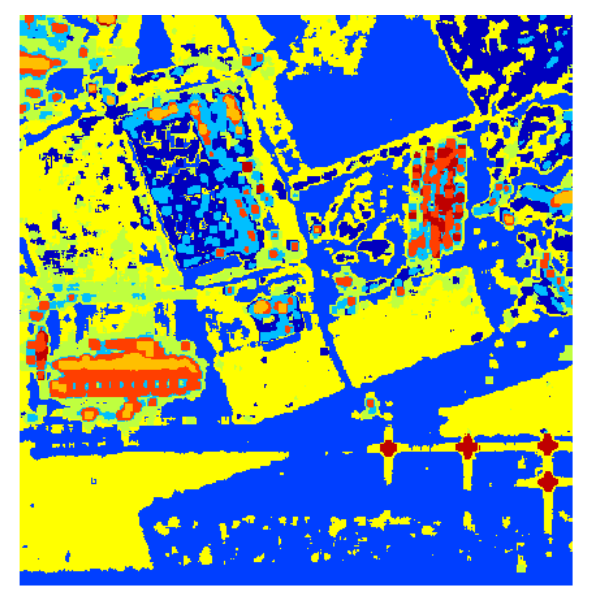

(a)

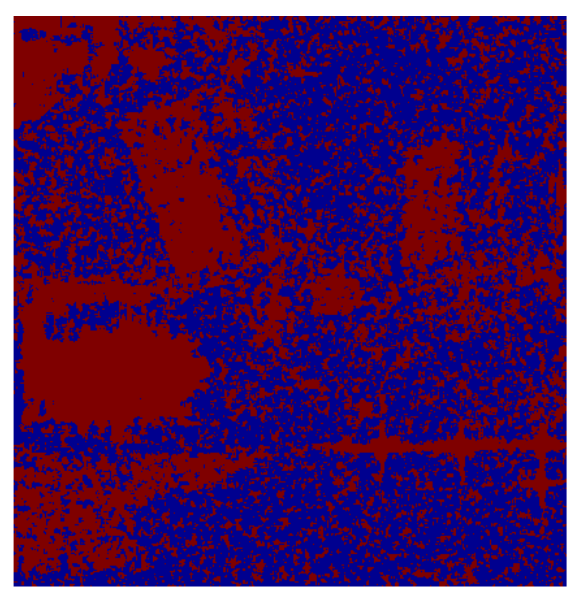

(b)

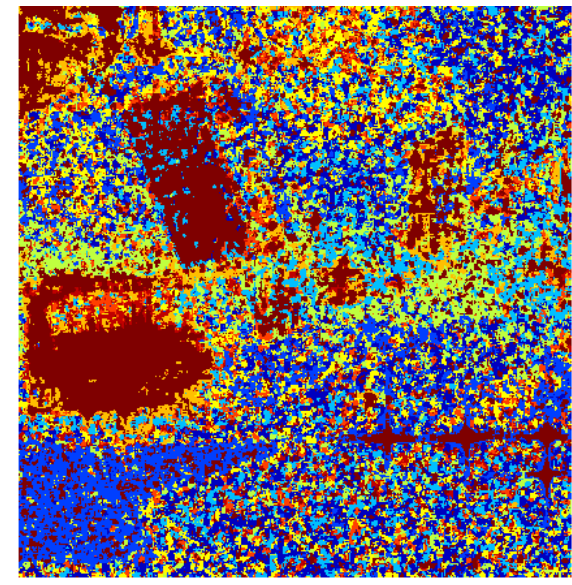

(c)

Fig. 2. Results of the Wishart Classifier and of the classifier using the M-distance

[2] F. Gini and M.V. Greco, "Covariance matrix estimation for CFAR detection in correlated heavy-tailed clutter," 
Signal Processing, vol. 82, no. 12, pp. 1847-1859, 2002.

[3] Frédéric Pascal, Yacine Chitour, Jean-Philippe Ovarlez, Philippe Forster, and P. Larzabal, "Covariance structure maximum-likelihood estimates in compound gaussian noise : Existence and algorithm analysis," IEEE Trancactions on Geoscience and Remote Sensing, vol. 56, no. 1, pp. 34-48, 2008.

[4] Frédéric Pascal, Jean-Philippe Ovarlez, Philippe Forster, and P. Larzabal, "Performance analysis of covariance matrix estimates in impulsive noise," Signal Processing, vol. 56, no. 6, pp. 2206-2216, 2008.

[5] Jong-Sen Lee, Mitchell R. Grunes, Thomas L. Ainsworth, Li-Jen Du, Dale L. Schuler, and Shane R. Cloude, "Unsupervised classification using polarimetric decomposition and the complex Wishart classifier," IEEE Transactions on Geoscience and Remote Sensing, vol. 37, no. 5, pp. 2249-2258, September 1999.

[6] G.E.P. Box, "A general distribution theory for a class of likelihood criteria," Biometrika, vol. 36, pp. 317.

[7] Knut Conradsen, Allan Aasbjerg Nielsen, Jesper Schou, and Henning Skriver, "A test statistic in the complex wishart distribution and its application to change detection in polarimetric sar data," IEEE Transactions on Geoscience and Remote Sensing, vol. 51, no. 1, pp. 4-19, January 2003. 